\title{
Moving Media and Frames in Acoustic Doppler Effect
}

\author{
Farrin Payandeh \\ Department of Physics, Payame Noor University (PNU), P.O. BOX, 19395-3697 Tehran, Iran
}

\section{Email address:}

Payandehfarrin92@gmail.com

\section{To cite this article:}

Farrin Payandeh. Moving Media and Frames in Acoustic Doppler Effect. American Journal of Mechanics and Applications. Vol. 3, No. 1, 2015, pp. 1-7. doi: 10.11648/j.ajma.20150301.11

\begin{abstract}
The Austrian Wuhan Doppler (1803-1853) revealed in a paper in 1842 [1] that the color of a luminous substance should change due to its relative movement and observer's. This effect which is named Doppler Effect can be seen through all the waves, and Doppler himself impressed on its application in acoustic waves. In 1845 an empirical experiment was performed using a moving truck carrying few people blowing trumpets. In this paper, the Doppler Effect has been investigated for moving observer, source, and media while they are moving in a straight line with together, and moving with an angle between the sound wave and observer's moving direction.
\end{abstract}

Keywords: Doppler Effect, Moving Media, Moving Source, Moving Frames, Acoustics

\section{Introduction: The Doppler Effect for Sound Waves}

If a sound wave source and an observer (who hears the sound) have a relative motion, then the frequency which is observed by the observer depends on the direction of the source's motion and therefore it defers from the original frequency. Using the Doppler Effect formulation [2-9], one can justify the relation between the observed and emitted frequencies. In this paper, we investigate the Doppler effect, in three general categories; first, when the observer and the source are located on a reference frame and are alone a straight line, second, when they are on different reference frames having relative motions, and third, when they are not located along a straight line; their velocities make an angle with the direction of sound emission.

\section{First Category}

Let us consider that the sound source is running toward the observer.

\subsection{First Case}

If the transferring medium is moving with a velocity $v_{m}$ from the source $\mathrm{S}$ to the observer $\mathrm{O}$, then we are dealing with two cases:

a)The source is stationary and the observer is running toward the source. In this case the wave crests are separated by a wavelength $\lambda$. The sound speed with respect to the medium is $v$. Clearly if the observer is supposed to be stationary in the medium, the number of wave crests which are detected by $\mathrm{O}$ within the time $t$ are

$$
n_{1}=\frac{v+v_{m}}{\lambda} t
$$

In this case, the sound is transferred by a velocity $v+v_{m}$, however since the observer is moving by a velocity $v_{0}$ toward the source, hence it observers some more crests by a number of $n_{2}=\frac{v_{0}}{\lambda} t$. The observed frequency would be

$$
f_{o}=\frac{\left(v+v_{m}\right)+v_{0}}{\lambda} .
$$

The emitted wavelength is

$$
\lambda=\frac{v+v_{m}}{f_{s}}
$$

Substituting (3) in (2) yields

$$
f_{o}=\frac{\left(v+v_{m}\right)+v_{0}}{\left(v+v_{m}\right)} f_{s}
$$

For a receding observer we have

$$
f_{o}=\frac{\left(v+v_{m}\right)-v_{0}}{\left(v+v_{m}\right)} f_{s} .
$$


b)The observer is stationary and the source is moving. As before, the same statements hold, therefore

$$
f_{o}=\frac{\left(v+v_{m}\right)}{\left(v+v_{m}\right) \mp v_{s}} f_{s}
$$

Therefore the general relation for frequency which is observed in $\mathrm{O}$ would be

$$
f_{o}=\frac{\left(v+v_{m}\right) \mp v_{o}}{\left(v+v_{m}\right) \mp v_{s}} f_{s} .
$$

The plus sign in the numerator and the minus sign in the denominator hold for the case in which observer and source are running towards, whereas the other case corresponds to when they are receding form.

\subsection{Second Case}

If the transferring medium is moving by a velocity $v_{m}$ in a reference frame from source to the observer, then the general relation changes to

$$
f_{o}=\frac{\left(v-v_{m}\right) \pm v_{o}}{\left(v-v_{m}\right) \mp v_{s}} f_{s} .
$$

\subsection{Third Case}

If the transferring medium and the reference frame are at rest with respect to each other, then $v_{m}=0$ and

$$
f_{o}=\frac{v \pm v_{o}}{v \mp v_{s}} f_{s}
$$

\section{Second Category}

In this section we assume that the source and the observer are located in their own peculiar moving frames; like roofless wagons. So in addition to the relative motion of source and observer, the wagons have their own relative motion as well. Let us notate the velocity of the source's wagon by $v_{s}^{\prime}$ and the on for observer by $v_{o}^{\prime}\left(v_{s}^{\prime}<v_{s}\right.$ and $\left.v_{o}^{\prime}<v_{o}\right)$.

\subsection{First Case}

In this case the transferring medium is moving by a velocity $v_{m}$ from the source to the observer.

a)Let the observer be moving and the source be at rest. Firstly we assume that the source and the wagon on which the source is located are at rest; i.e. $v_{s}=v_{s}^{\prime}=0$. Now if the observer moves by a velocity $v_{o}$ on its own wagon toward the source, two cases will arise:

1 . When the wagon moves by a velocity $v_{o}^{\prime}$ in the same direction of observer's motion toward the source, then the relative velocity of observer and source is $v_{o}+v_{o}^{\prime}$. Therefore the observer and the source are always approaching and hence, one can exploit the same relations as before to find

$$
\begin{aligned}
& f_{o}=\frac{\left(v+v_{m}\right)+v_{o}}{\left(v+v_{m}\right)} f_{s} \\
& \rightarrow f_{o}=\frac{\left(v+v_{m}\right)+\left(v_{o}+v_{o}^{\prime}\right)}{\left(v+v_{m}\right)} f_{s} .
\end{aligned}
$$

2.When wagon is moving in the opposite direction to the observer's motion and recedes from the source, the observer's and source's relative velocity is $v_{o}-v_{o}^{\prime}$. According to the fact that $v_{o}^{\prime}<v_{o}$, the net motion of the observer is towards the source. Therefore substituting $v_{o}-v_{o}^{\prime}$ for $v_{o}$, the observed frequency is calculated as follows:

$$
\begin{aligned}
& f_{o}=\frac{\left(v+v_{m}\right)+v_{o}}{\left(v+v_{m}\right)} f_{s} \\
& \rightarrow f_{o}=\frac{\left(v+v_{m}\right)+\left(v_{o}-v_{o}^{\prime}\right)}{\left(v+v_{m}\right)} f_{s} .
\end{aligned}
$$

Now if we assume that the observer is moving by a velocity $v_{o}$ on its own wagon, receding from source, then we have again two cases.

1. When the wagon is moving by a velocity $v_{o}^{\prime}$ toward the source, then the relative velocity between observer and the source will be $v_{o}-v_{o}^{\prime}$. However according to $v_{o}^{\prime}<v_{o}$, the observer is generally receding from the source. Therefore to obtain $f_{o}$, we use same relations as they are in the previous section. So

$$
\begin{aligned}
& f_{o}=\frac{\left(v+v_{m}\right)-v_{o}}{\left(v+v_{m}\right)} f_{s} \\
& \rightarrow f_{o}=\frac{\left(v+v_{m}\right)-\left(v_{o}-v_{o}^{\prime}\right)}{\left(v+v_{m}\right)} f_{s} .
\end{aligned}
$$

2. When the Wagon is receding from the source by a velocity $v_{o}^{\prime}$, then one can conclude

$$
\begin{aligned}
& f_{o}=\frac{\left(v+v_{m}\right)-v_{o}}{\left(v+v_{m}\right)} f_{s} \\
& \rightarrow f_{o}=\frac{\left(v+v_{m}\right)-\left(v_{o}+v_{o}^{\prime}\right)}{\left(v+v_{m}\right)} f_{s} .
\end{aligned}
$$

Therefore the general relation for the frequency conversion in these cases is

$$
f_{o}=\frac{\left(v+v_{m}\right) \pm\left(v_{o} \pm v_{o}^{\prime}\right)}{\left(v+v_{m}\right)} f_{s} .
$$

b) The observer is at rest and the source is moving. Now let us assume that the observer and its own wagon are both at rest $\left(v_{o}=v^{\prime}{ }_{o}=0\right)$. If the source is moving by a velocity $v_{s}$ on its own wagon toward the observer, we will have two cases: 
1. When the wagon is moving with the velocity $v_{s}^{\prime}$ in the same direction of the source's motion toward the observer, then the net velocity of source is $v_{s}+v^{\prime}{ }_{s}$ and hence, to obtain $f_{o}$ we have

$$
\begin{aligned}
& f_{o}=\frac{\left(v+v_{m}\right)}{\left(v+v_{m}\right)-v_{s}} f_{s} \\
& \rightarrow f_{o}=\frac{\left(v+v_{m}\right)}{\left(v+v_{m}\right)-\left(v_{s}+v_{s}^{\prime}\right)} f_{s} .
\end{aligned}
$$

2. When the wagon is moving apart from the observer with the previous velocity, then the relative velocity between source and observer is $v_{s}-v_{s}^{\prime}$ and since $v_{s}^{\prime}<v_{s}$ then the net motion is approaching to the observer. So

$$
\begin{aligned}
& f_{o}=\frac{\left(v+v_{m}\right)}{\left(v+v_{m}\right)-v_{s}} f_{s} \\
& \rightarrow f_{o}=\frac{\left(v+v_{m}\right)}{\left(v+v_{m}\right)-\left(v_{s}-v_{s}^{\prime}\right)} f_{s} .
\end{aligned}
$$

Now let us assume that the source is moving on its own wagon in opposite direction to the former case. Here we also confront two cases:

1. When the wagon is moving toward the observer, then the relative velocity becomes $v_{s}-v_{S}^{\prime}$ and since $v_{s}>v_{s}^{\prime}$, then the net motion is a recession and therefore

$$
\begin{aligned}
& f_{o}=\frac{\left(v+v_{m}\right)}{\left(v+v_{m}\right)+v_{s}} f_{s} \\
& \rightarrow f_{o}=\frac{\left(v+v_{m}\right)}{\left(v+v_{m}\right)+\left(v_{s}-v_{s}^{\prime}\right)} f_{s} .
\end{aligned}
$$

2. When the wagon, in the same direction of source's motion, is receding from observer, then again we have a recession and

$$
\begin{aligned}
& f_{o}=\frac{\left(v+v_{m}\right)}{\left(v+v_{m}\right)+v_{s}} f_{s} \\
& \rightarrow f_{o}=\frac{\left(v+v_{m}\right)}{\left(v+v_{m}\right)+\left(v_{s}-v_{s}^{\prime}\right)} f_{s} .
\end{aligned}
$$

According to above considerations, the general relation for frequency shift is

$$
f_{o}=\frac{\left(v+v_{m}\right)}{\left(v+v_{m}\right) \mp\left(v_{s} \pm v_{s}^{\prime}\right)} f_{s} .
$$

\subsection{Second Case}

In all mentioned cases, if the transferring medium is moving by a velocity $v_{m}$ with respect to the reference frame, from the observer to the source, it is sufficient to use $v-v_{m}$ instead of $v+v_{m}$ in above relations.

\section{Third Category}

In this section, we consider a situation in which the direction of the source's motion and observer's motion in the reference frame, make an angle $\alpha$ with the direction of the sound which is coming from the source.

Now if the transferring medium is moving by a velocity $v_{m}$ relative to the reference frame, from left to right.

a)When the observer is moving while the source is at rest, we assume that the source is located at

a point like $\mathrm{P}$ (See figure 1 below).

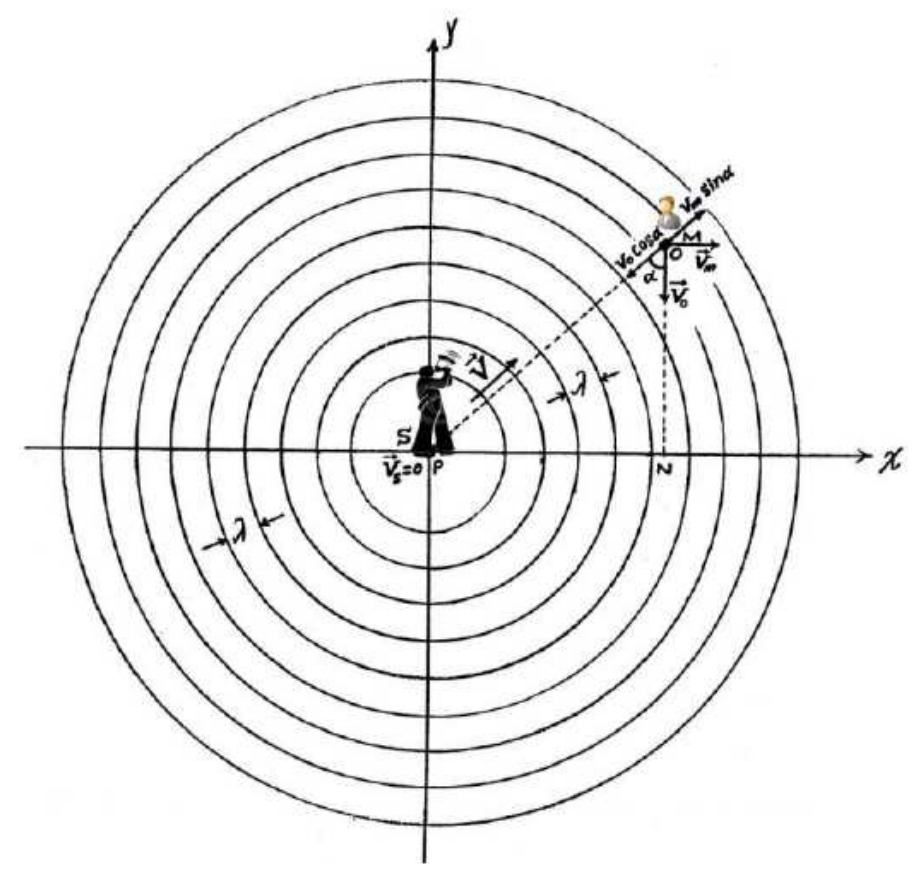

Figure 1. Observer is moving while the source is at rest 
According to the figure, $v_{s}=0$ and the observer $\mathrm{O}$ is moving along $\mathrm{MN}$ with a velocity $v_{o}$, in a direction orthogonal to $x$ axis from $\mathrm{M}$ to $\mathrm{N}$. If the angle between the direction of observer's motion and the direction of sound propagation is $\alpha(\angle \mathrm{PMN}=\alpha)$, then the observer's velocity along $\mathrm{MN}$ is $v_{o}$ and the velocity of transferring medium, according to the figure, is $v_{m}$. The distance between observer at $\mathrm{M}$ and the source at $\mathrm{P}$ is $\mathrm{MP}$, whereas this distance from N to P is NP. On the other hand, since MP is the hypotenuse of $\triangle \mathrm{MPN}$. Hence, MP $>$ NP, i.e. when observer is moving from $\mathrm{M}$ to $\mathrm{N}$, its distance to the source is gradually decreasing.

If the observer is at rest at $\mathrm{M}$, according to the fact that the component of velocity of transferring medium along MP is $v_{m} \sin \alpha$ in the direction of sound propagation, therefore the number of wave crests reaching $\mathrm{O}$ in a time interval $t$ is

$$
n_{1}=\frac{\left(v+v_{m} \sin \alpha\right)}{\lambda} t
$$

However, the observer is moving orthogonally to $x$ axis by the velocity $v_{o}$. Therefore the number of wave crests which is received by $\mathrm{O}$ while moving from $\mathrm{M}$ to $\mathrm{N}$ is altered by $n_{2}=\frac{\left(v_{o} \cos \alpha\right)}{\lambda} t$. According to figure 1 , it is evident that during this displacement, $\alpha$ is continually increasing and consequently, $n_{2}$ is decreasing. The general number of wave crests is therefore

$$
\begin{aligned}
& n=n_{1}+n_{2} \\
& =\frac{\left(v+v_{m} \sin \alpha\right)}{\lambda} t+\frac{v_{o} \cos \alpha}{\lambda} t .
\end{aligned}
$$

However, since $\alpha$ experiences this increase during observer's motion form $\mathrm{M}$ to $\mathrm{N}$, therefore although the observer is gradually approaching the source, the number of receiving wave crests will decrease, and

$$
f_{o}=\frac{n}{t}
$$

Substituting $n$ from (21) in (22) yields

$$
f_{o}=\frac{\left(v+v_{m} \sin \alpha\right)+v_{o} \cos \alpha}{\lambda} .
$$

Also substitution of the value of $\lambda$ gives

$$
\begin{aligned}
& \lambda=\frac{\left(v+v_{m} \sin \alpha\right)}{f_{s}} \\
& \rightarrow f_{o}=\frac{\left(v+v_{m} \sin \alpha\right)+v_{o} \cos \alpha}{\left(v+v_{m} \sin \alpha\right)} f_{s} .
\end{aligned}
$$

The above relation shows that along observer's motion and by increasing $\alpha$, the observed frequency $f_{o}$ will decrease. Now, according to the figure 2 , we assume that $\mathrm{O}$ is approaching $\mathrm{L}$ along NL by a velocity $v_{o}$, orthogonally to the $x$ axis.

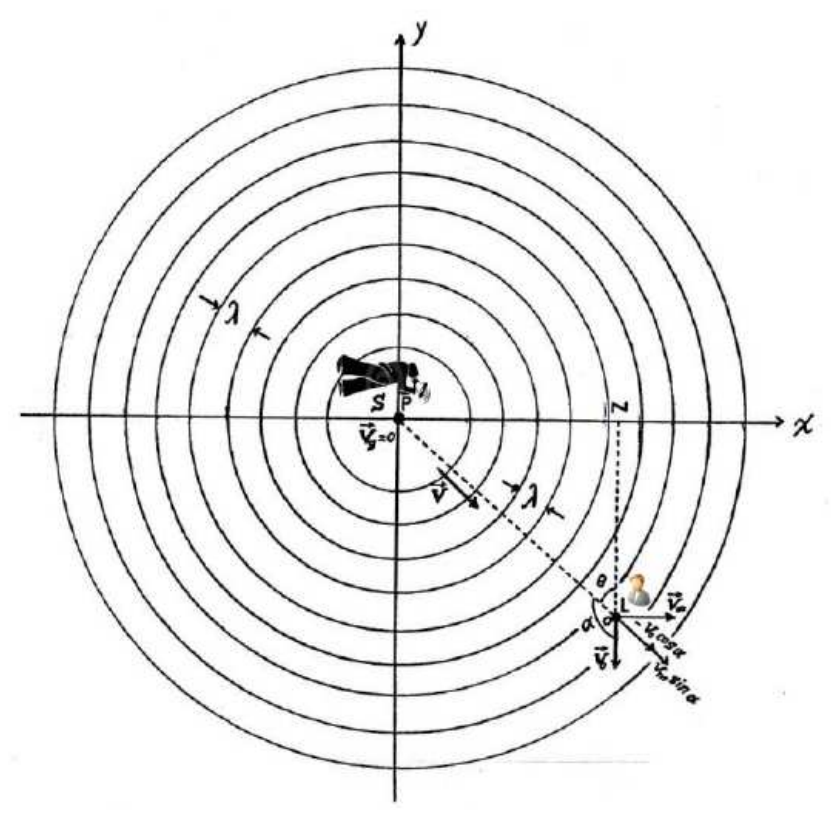

Figure 2. The observer is moving by a relative angle $\alpha$

In this case also, the angle between the direction of observer's motion and the emitted sound is supposed to be $\alpha$. Moreover, since LP is the hypotenuse of ${ }_{\triangle \mathrm{NLP}}$, therefore
LP $>$ NL and the observer is receding from source while moving along NL.

As it is pointed out from figure 2, in this case of observer's 
relative motion to the static source, the components of the transferring medium velocity and observer's velocity along LP, are in the same direction of emitted sound and are respectively $v_{m} \sin \alpha$ and $-v_{o} \cos \alpha$. Since the observer is receding from the source, the number of observed wave crests is altered by

$$
n_{2}^{\prime}=\frac{\left(-v_{o} \cos \alpha\right)}{\lambda} t
$$

Also since $\alpha$ is increasing during this motion, and since $\alpha$ is always greater than $90^{\circ}$, therefore increase in this angle may lead to an increase in the component of observer's velocity along PL (i.e. $-v_{o} \cos \alpha$ ) and consequently, to an increase in $n_{2}^{\prime}$. However, the net number of observed wave crests will inevitably decrease.

$$
\begin{aligned}
& n=n_{1}+n_{2}^{\prime} \\
& =\frac{\left(v+v_{m} \sin \alpha\right)}{\lambda} t-\frac{\left(-v_{o} \cos \alpha\right)}{\lambda} t .
\end{aligned}
$$

However according to the fact that during the whole motion $\alpha$ is always increasing, therefore from (26) one may conclude that the number of observed wave crests $(n)$, during motion from $\mathrm{N}$ to $\mathrm{L}$ will decrease and becomes

$$
\begin{aligned}
& f_{o}=\frac{n}{t} \\
& \rightarrow f_{o}=\frac{\left(v+v_{m} \sin \alpha\right)+\left(v_{o} \cos \alpha\right)}{\lambda} .
\end{aligned}
$$

On the other hand we have

$$
\lambda=\frac{\left(v+v_{m} \sin \alpha\right)}{f_{s}}
$$

Substituting (28) in (27) we have

$$
f_{o}=\frac{\left(v+v_{m} \sin \alpha\right)+\left(v_{o} \cos \alpha\right)}{\left(v+v_{m} \sin \alpha\right)} f_{s} .
$$

As we can see, relation (29) is perfectly the same as (24) and therefore points out that in this case of relative motion between observer and the static source, for observer's motion from $\mathrm{N}$ to $\mathrm{L}$, the observed frequency $f_{o}$ is always decreasing while $\alpha$ is increasing. The equality between (24) and (29) also asserts that, when the observer is moving from $\mathrm{G}$ to $\mathrm{L}$, orthogonally to $x$ axis, firstly it approaches the source and then recedes from it. The observed frequency for both cases is given by same relations and this frequency, depending on the increase in $\alpha$, always decrease and generally is given by (29). Note that the observed frequency while the observer is moving along GL, has its maximum at G. For $\alpha=90^{\circ}$, this frequency becomes the same as the emitted one. Then for $0<\alpha<90^{\circ}$ the observed frequency becomes greater than the original one. However during the observer's recession from the source, we have $90^{\circ}<\alpha<180^{\circ}$ and the observed frequency is always lesser than the original one.

b) When the observer is at rest and the source is moving. According to figure 3, we assume that the observer $\mathrm{O}$ is at rest at the point $\mathrm{N}\left(v_{o}=0\right)$ and the source $\mathrm{S}$ is moving along HP orthogonally to $x$ axis by a velocity $v_{s}$ from $\mathrm{H}$ to $\mathrm{P}$.

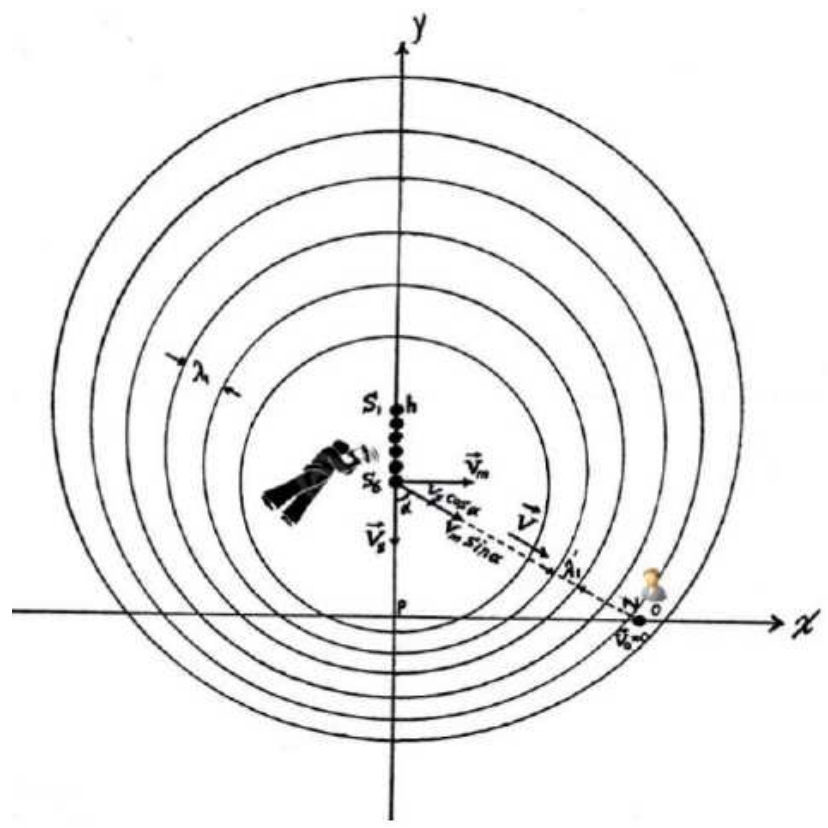

Figure 3. The observer is at rest and the source is moving towards by a relative angle

If $\alpha$ is the angle between the direction of source's motion and the observed frequency by the observer (i.e. $\angle \mathrm{PHN}=\alpha)$, then the component of the source's velocity along $\mathrm{HN}$ is $v_{s} \cos \alpha$ and the component of the transferring medium's velocity along the same direction is $v_{m} \sin \alpha$. 
Since NH is the hypotenuse of $\triangle \mathrm{PHN}$, always $\mathrm{HN}>\mathrm{PN}$. This means that when the source is moving from $\mathrm{H}$ to $\mathrm{P}$ along $\mathrm{HP}$, its distance to the observer is gradually decreasing. In this process, if the source is at rest at $\mathrm{H}$, since the component of transferring medium velocity along $\mathrm{HN}\left(v_{m} \sin \alpha\right)$ is in the same direction of the emitted sound from source, therefore the observed wavelength by the static observer, is equal to

$$
\lambda=\frac{\left(v+v_{m} \sin \alpha\right)}{f_{s}} .
$$

Hence, in this case of moving source from $\mathrm{H}$ to $\mathrm{P}$, the observed frequency is altered by $\lambda_{1}=\frac{\left(v_{s} \cos \alpha\right)}{f_{s}}$. During source's motion since $\alpha$ experiences an increase, so $\lambda_{1}$ decreases. Therefore the observed wavelength is

$$
\begin{aligned}
& \lambda_{o}=\lambda-\lambda_{1} \\
& =\frac{\left(v+v_{m} \sin \alpha\right)}{f_{s}}-\frac{\left(v_{s} \cos \alpha\right)}{f_{s}} .
\end{aligned}
$$

However according to figure $3, \alpha$ increases whilst source is moving from $\mathrm{H}$ to $\mathrm{P}$ and therefore $\lambda_{o}$ increases because of (31), despite the approach of the source. Therefore the observed frequency is obtained by substituting (31) into (30).

$$
f_{o}=\frac{\left(v+v_{m} \sin \alpha\right)}{\left(v+v_{m} \sin \alpha\right)-\left(v_{s} \cos \alpha\right)} f_{s} .
$$

As it is apparent from (32), increasing $\alpha$ leads to decreasing the observed frequency. Therefore while the source is moving towards the observer, the observed frequency becomes gradually lesser.

Now turning to the figure below, we assume that the source is moving along $\mathrm{PK}$, orthogonally to the $x$ axis, form $\mathrm{P}$ to $\mathrm{K}$.

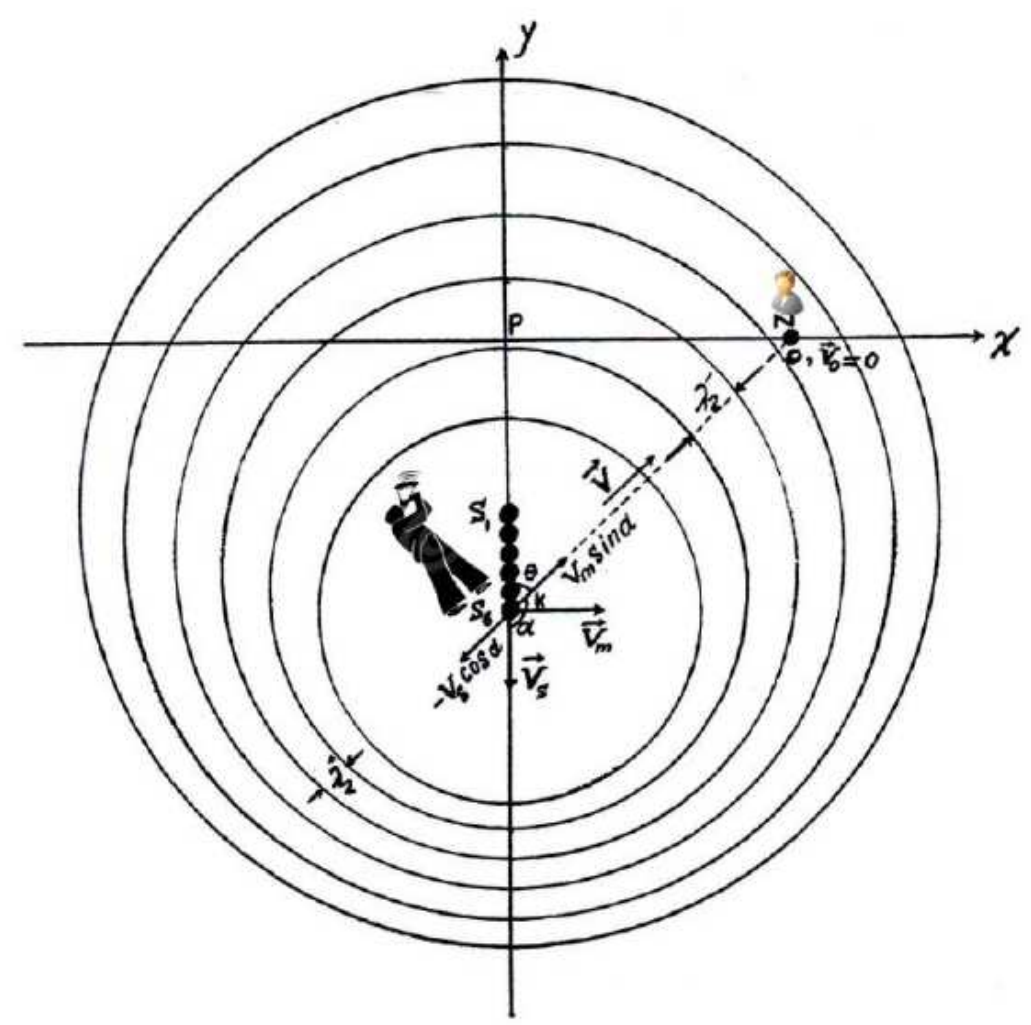

Figure 4. Showing the components of source's and the transferring media's velocities

In this case as well as before, the angle between the direction of source's motion and the received sound is named $\alpha$. Since $\mathrm{KN}$ is the hypotenuse of $\triangle \mathrm{PKN}$, then always $\mathrm{KN}>\mathrm{PN}$; i.e. the source is always receding from observer. Figure 4 shows explicitly that the component of source's and the transferring media's velocities along $\mathrm{KN}$, are respectively $-v_{s} \cos \alpha$ and $v_{m} \sin \alpha$. Therefore within source's motion from $\mathrm{P}$ to $\mathrm{K}$, the observed wavelength is altered by $\lambda_{2}=\frac{\left(-v_{s} \cos \alpha\right)}{f_{s}}$. Increasing $\alpha$, the component of sources velocity along $\mathrm{KN}$ will increase. Hence, the amount of change in the observed wavelength $\left(\lambda_{2}\right)$ will also increase. Consequently, the net observed wavelength becomes

$$
\begin{aligned}
& \lambda_{o}=\lambda+\lambda_{2} \\
& =\frac{\left(v+v_{m} \sin \alpha\right)}{f_{s}}+\frac{\left(-v_{s} \cos \alpha\right)}{f_{s}} .
\end{aligned}
$$

However it is known that during source's motion form $\mathrm{P}$ to 
$\mathrm{K}, \alpha$ is always increasing and is also the changes in $\lambda_{2}$. Therefore in general, the observed wavelength, depending on the increase in $\alpha$, will also increase. Hence, substituting the value for $\lambda$ in (33), the observed frequency becomes

$$
f_{o}=\frac{\left(v+v_{m} \sin \alpha\right)}{\left(v+v_{m} \sin \alpha\right)-\left(v_{s} \cos \alpha\right)} f_{s} .
$$

As it is seen, (34) and the formerly obtained relation (29) are the same. This equality states that, for the case of static observer and moving source, as well as the case of moving observer and static source, an increase in $\alpha$ results in a decrease in observed frequency. This equality also shows that, when the source is moving along HK, orthogonally to the $x$ axis from $\mathrm{H}$ to $\mathrm{K}$, the observed frequency in both cases of approaching and receding source is given by same relations, i.e. equation (34). According to the above statements about the source's motion toward the static observer, and regarding figures 1 and 2, during source's motion along HK relative to the static observer, when the source is located instantly at $\mathrm{H}$, the observed frequency is greater than the case in which the source is located at $\mathrm{K}$. Therefore this frequency is decreasing while the source is moving from $\mathrm{H}$ to $\mathrm{K}$, and this is because the increase in $\alpha$. Also for the instant in which $\alpha=90^{\circ}$, the observed frequency is the same as the originally emitted one. It is worth mentioning that when the source is approaching the static observer, the angle $\alpha$ resides in the interval $0<\alpha<90^{\circ}$ and the observed frequency is greater than the emitted one. However when the source is receding from the static observer, we have $90^{\circ}<\alpha<180^{\circ}$, and the observed frequency is lesser than the emitted one. Therefore the general relation for the observed frequency is obtained by reconciling (33) and (24) yielding

$$
f_{o}=\frac{\left(v+v_{m} \sin \alpha\right)+\left(v_{o} \cos \alpha\right)}{\left(v+v_{m} \sin \alpha\right)-\left(v_{s} \cos \alpha\right)} f_{s} .
$$

\section{Summary}

In this paper, we paid attention to the Doppler effect, in diversified categories including both static and moving observers and sources, and also the cases in which their motion and the direction of observed sound make angles. We demonstrated how these cases may reflect in the value of the observed frequencies. Also some special examples in each case were given.

\section{References}

[1] C.J. Doppler: .About the colored light of the binary stars and some other stars of the heavens, Proceedings of the Royal Bohemian Society of Sciences (Part V, Vol 2), Prague, (1842)

[2] D. Halliday, R. Resnick, J. Walker: Fundamentals of Physics, John Wiley and Sons; 7th edition (June 14, 2004), ISBN-13: 978-0471216438

[3] A. Eden: The search for Christian Doppler, Springer-Verlag, Wien (1992)

[4] E. Adrian: Doppler Effect, NCSA (1995)

[5] B. Ballot: Annalen der Physik und Chemie 11: 321-351 (1845)

[6] Fizeau: Acoustique et optique, Lecture, Société Philomathique de Paris, 29 December, (1848)

[7] J. S. Russell: Report of the Eighteenth Meeting of the British Association for the Advancement of Science, London, 18, 7, 37-38 (1849)

[8] J. Rosen, L.Q. Gothard: Encyclopedia of Physical Science. Infobase Publishing. p. 155 (2009)

[9] J. William: The Theory of Sound 2 (2 ed.), MacMillan \& Co, p. $154(1896)$ 